\title{
Diagnóstico del Sistema de Control Interno en la Fundación vida nueva y digna de la ciudad de Montería
}

\section{Diagnosis of the Internal Control System at the new and digna life Foundation of the city of Montería}

\author{
Carlos A. Causil-Lengua* \\ Corporación Universitaria Americana - Colombia \\ ORCID iD: https://orcid.org/0000-0001-6685-1201 \\ causilcarlos99@americana.edu.co
}

\section{Alexander Bravo-Yepes \\ Corporación Universitaria Americana - Colombia ORCID iD: https://orcid.org/0000-0002-7756-423X ayepes@coruniamericana.edu.co}

\section{Jhennys P. Becerra-Ossa \\ Corporación Universitaria Americana - Colombia \\ ORCID iD: https://orcid.org/0000-0003-0732-4721 \\ jbecerra@coruniamericana.edu.co}

* Autor a quien debe ser dirigida la correspondencia
Fecha de recepción: 01/10/2021

Fecha de evaluación: 17/10/2021

Fecha de aceptación: 04/11/2021

Cómo citar: Causil-Lengua, C., Bravo-Yepes, A., \& Becerra-Ossa, J. (2021). Diagnóstico del Sistema de Control Interno en la Fundación vida nueva y digna de la ciudad de Montería. Revista Cientifica Anfibios, 4(2). https://doi.org/10.37979/afb.2021v4n2.90

\section{Resumen}

La presente investigación se centró en realizar un diagnóstico del Sistema de Control Interno (SCI) en la Fundación Vida Nueva y Digna de la ciudad de Montería, utilizando para ello el modelo de control interno COSO. La unidad muestral corresponde a la Fundación, tomando para el estudio 12 sujetos participantes de los cargos gerenciales, administrativos, operativos y órganos de control que se encontraban relacionados directamente con las actividades de control; los instrumentos utilizados para la recolección de datos fueron un cuestionario que contenía 18 ítems y una lista de verificación o chequeo, empleada con el fin de ampliar la información obtenida a través del cuestionario aplicado. Como resultado de la investigación se pudo obtener que la organización objeto de estudio se encuentra expuesta a diversos riesgos de índole financiero, económico y legal, toda vez que existen fallas en la implementación y estructuración del Sistema de Control Interno, donde se pudo evidenciar que no existe un área designada o encargada del manejo del mismo, lo cual afecta la independencia de un proceso frente a otro al momento de establecer los controles y realizar las medidas preventivas y/o correctivas a que hubiese lugar. El Sistema de Control Interno es una herramienta esencial en este tipo de organizaciones porque ayuda a minimizar riesgos y realizar una mejor toma de decisiones, obteniendo como resultado una mayor operacionalidad y flujo en los procesos.

\section{Palabras clave}

Control interno; ESAL; diagnóstico; procesos y procedimientos; políticas

\begin{abstract}
This research focused on carrying out a diagnosis of the Internal Control System at the New and Digna Life Foundation of the city of Montería, using the COSO internal control model. The sample unit corresponds to the Foundation, taking for the study 12 subjects participating in the managerial, administrative, operational and control positions that were directly related to the control activities; the instruments used for data collection were a questionnaire containing 18 items and a check or checklist, used to expand the information obtained through the questionnaire applied. As a result of the investigation, it was possible to obtain that the organization under study is exposed to various risks of a financial, economic and legal nature, since there are failures in the implementation and structuring of the Internal Control System, where it could be evidenced that no There is a designated or
\end{abstract}


responsible area for managing it, which affects the independence of one process from another when establishing controls and carrying out the preventive and / or corrective measures that may take place. The Internal Control System is an essential tool in this type of organizations because it helps minimize risks and make better decision-making, resulting in greater operationality and process flow.

\section{Keywords}

Internal control; non-profit entity; diagnosis; processes and procedures; policies

\section{Introducción}

En un mundo globalizado como el de hoy, las organizaciones cada día sin importar su naturaleza jurídica o su objeto social intentan obtener la mayor confiabilidad en sus operaciones y en los sistemas de información que adoptan e implementan. Todos los cambios que ha traído consigo el fenómeno de la globalización afectan de forma directa el actuar de las organizaciones generando de esta manera un mayor interés por alcanzar un alto grado de competitividad en el mercado y el sector en que se desarrollan, teniendo en cuenta que "La globalización mejora, en conjunción con la nueva ola de desarrollo de las tecnologías, la eficiencia de la economía en general y, por tanto, la productividad y el crecimiento mundial" (De la Dehensa, 2000, p.165). Esto demuestra que las compañías, entidades u organizaciones, ya sean de carácter público o privado están siendo forzadas a medir e impulsar sus actividades de acuerdo a las características que presentan los nuevos mercados económicos.

Con el fin de tener control y evaluar en la mayor medida posible los procesos que se desarrollan en cada una de las áreas o dependencia de las empresas, estas optan por la implementación de un Sistema de Control Interno (SCI) donde todos los integrantes de la organización son responsables de la efectividad y el buen funcionamiento de este, aunque es evidente que hay un mayor involucramiento y responsabilidad en los niveles superiores. Es importante reconocer que el funcionamiento de esta herramienta va a depender de las políticas y parámetros establecidos por la organización, a pesar de que existen ciertos modelos de control interno, no existe una regulación para la implementación de los mismos, lo cual le da la libertad a las organizaciones de escoger el que crea conveniente y realizar los respectivos ajustes, adaptándolo a las necesidades $\mathrm{y}$ proyectos institucionales, teniendo en cuenta igualmente que "Ahora es mayor la exposición a los riesgos de fraude que lo que ocurría hace dos o tres décadas atrás; por ello es necesario invertir mucho más ahora en controles para prevenir y detectar fraude" (Aguiar,1996, p.204). Lo anterior, indica que los controles deben ser una medida de prevención más que de corrección y aunque en ciertos momentos es difícil la detección de riesgos y fraudes, con la implementación de estos se minimizan las fallas en los procesos y procedimientos.

Por otro lado, en el caso de las entidades sin ánimo de lucro (ESAL) la adopción e implementación de un SCI tiene la misma finalidad que pudiese tener para cualquier otra organización. En este sentido, se plantea:

Las empresas con ánimo o sin ánimo de lucro deben propender a crear valor a sus protectores, dueños o accionistas, así como la de enfrentar y superar las incertidumbres, (...), poder proveer una estructura conceptual, (...), y así enriquecer su capacidad para generar valor (Estupiñán, 2015, p. 25).

Esto indica, que es necesario entender que su buen funcionamiento ayudará a alcanzar los objetivos empresariales; debido a esto, es de vital importancia desarrollar un monitoreo y seguimiento constante para obtener así una mayor efectividad y eficacia en la ejecución de los procesos administrativos, financieros y contables, lo cual genera una mayor credibilidad y competitividad. El impuesto a las ventas o Impuesto al Valor Agregado comúnmente denominado IVA, es un tributo que tiene su efecto en el consumo de servicios, bienes y explotación de juegos de azar y suerte. (Villasmil \& Polo, 2018).

Se realizará un diagnóstico del Sistema de Control Interno en la Fundación Vida Nueva y Digna de la ciudad de Montería, de tal forma que se pueda determinar el nivel de conocimiento que poseen los funcionarios de las áreas que conforman la fundación, en lo que refiere a la importancia, objetivos y buen funcionamiento del SCI; además, se evaluará el SIC, en lo que 
concierne a la estructuración, documentación y funcionamiento, bajo los componentes del marco de control interno del COSO; y finalmente se propondrán una serie de recomendaciones para fortalecer el SIC y la toma de decisiones en los procesos administrativos, contables y financieros de la Fundación Vida Nueva y Digna.

\section{Método}

En la investigación se desarrolló un tipo de metodología o investigación descriptiva, teniendo en cuenta que "los estudios descriptivos son útiles para mostrar con precisión los ángulos o dimensiones de un fenómeno, suceso comunidad, contexto o situación" (Hernández, Fernández, y Baptista, 2010, p.80). Entonces, a través de esta metodología es posible conocer e identificar todos aquellos factores que se encuentran relacionados con la situación actual que presenta el control interno dentro de la organización objeto de estudio. Del mismo modo, entendiendo que el fenómeno se estudia y la recolección de la información se realiza partiendo de la observación sobre un grupo en particular, el método a través del cual se desarrolló el trabajo de investigación fue inductivo, con un enfoque mixto, el cual resulta de la unión y contempla los elementos característicos del método cualitativo y cuantitativo. El estudio de las teorías de desarrollo económico regional proporcionan ideas más amplias y concisas sobre las disparidades regionales presentes en un país, a diferencia de las teorías de desarrollo económico. (De la Cruz, 2018)

La población objeto de estudio en la cual se realizó el estudio corresponde a una sola entidad, la Fundación Vida Nueva y Digna. El tipo de muestreo seleccionado en la investigación corresponde a aquel denominado muestreo por conveniencia, toda vez que las personas seleccionadas que participan como informantes o "las unidades de la muestra se autoseleccionan o se eligen de acuerdo a su fácil disponibilidad" (Mejía, 2000, p. 169). En este sentido, la unidad muestral no fue elegida dentro de la población de acuerdo a una serie de criterios y/o características comunes, sino más bien de acuerdo a la facilidad y comodidad del investigador y a la disponibilidad de la unidad o unidades muestrales o de observación. Por tanto, el estudio investigativo abarcó a la Fundación como unidad muestral, escogiendo para ello 12 sujetos participantes.
Para llevar a cabo la investigación y teniendo en cuenta los procedimientos para la recolección de información sobre el fenómeno o suceso estudiado, se hizo uso de la encuesta y la lista de chequeo (Check List) o verificación como los instrumentos de recolección de datos válidos dentro del proceso investigativo. Además, se desarrolló un tipo de paradigma de análisis de investigación hermenéutico, el cual presenta como una de sus características principales "la identificación de las reglas que subyacen, siguen y gobiernan los fenómenos sociales" (Pérez, 1994, p.27). De esta manera, los datos e información obtenida estuvieron influidos por las características y el contexto en el cual se llevó a cabo el estudio. Así mismo, esta investigación se desarrolló teniendo en cuenta principios éticos tales como: imparcialidad, equidad, justicia y confidencialidad; y conforme a los diferentes criterios establecidos por la organización y la institución académica; y con la declaratoria de consentimiento informado de los sujetos participantes.

\section{Resultados}

\section{EI Sistema de Control Interno en las organiza- ciones}

Hablar de control interno, es hablar de gestión, planificación, y mecanismos de evaluación, que conllevan a la verificación de lo procedimental, garantizando la transparencia y el actuar del ente económico, vigilado por organismos Estatales; para los cuales establecer un buen sistema de control interno en los entes económicos, como es el caso de las ESAL, es una garantía del resguardo de los recursos, y evitar los posibles fraudes por negligencias administrativas. Los riesgos pertenecen a la naturaleza de cualquier negocio y nacen con el surgimiento del mismo, pero es allí donde cada organización marca la diferencia al implementar y establecer estrategias enfocadas a la minimización de estos.

El Sistema de control interno es una herramienta fundamental para la consecución de los objetivos trazados, donde todos los actores de la empresa tienen cierto grado de participación, el control interno busca en cierta manera ayudar a la gerencia a desarrollar una mejor gestión, análisis y evaluación de los procesos y procedimientos, con el fin de realizar una toma de decisiones en tiempo real y/u oportuno, permitiendo mejorar el 
alcance de los objetivos empresariales establecidos y la evaluación y administración de los riesgos; lo anterior lo apoya Mantilla (2008), quien define el control interno como "un proceso diseñado e implementado por la administración para tratar los riesgos del negocio y de fraude identificados que amenazan el logro de los objetivos establecidos, tales como la confiabilidad de la información financiera" (p.43). Se puede observar según lo descrito anteriormente por el autor que la implementación de un sistema de control interno dentro de una organización es fundamental porque nos ayuda a detectar las fallas que existan en los procesos internos que se llevan dentro de estas, facilitando de esta forma la toma de decisiones en el momento oportuno. Sin embargo, el tener un sistema de control interno establecido no necesariamente significa que esté siendo útil, ya que se deben realizar revisiones, evaluaciones y actualizaciones del mismo, de lo contrario van a salir a relucir deficiencias en esta herramienta que de cierta forma impactarán en el ejercicio y la toma de decisiones gerencial; para lo cual se debe procurar establecer un ambiente de control propicio para la eficacia y eficiencia del mismo. Arens, Elder y Beasley (2007) definen "La esencia de la organización controlada de forma eficaz está en la actitud de su administración" (p.275). Por consiguiente, tal como sea percibido el control desde los niveles superiores así se verá reflejado y se responderá en los niveles inferiores, esto implica la implementación y adopción de estrategias enfocadas al mejoramiento de esta herramienta, lo cual va a requerir de más esfuerzo y compromiso de las diferentes áreas y de la misma dirección administrativa.

\section{Nivel de conocimiento de los funcionarios con respecto a la importancia, objetivos y buen fun- cionamiento del Sistema de Control Interno}

En el marco de la evaluación e implementación del Sistema de Control Interno en cualquier organización, es necesario que todos sus actores o personas que se encuentran en las diversas áreas o departamentos tengan pleno conocimiento de cómo funciona y la importancia del mismo, ya que a partir de ello se puede analizar su eficacia y eficiencia para ayudar en la toma de decisiones, sin embargo, la investigación pudo arrojar que existen una serie de factores tales como la inexistencia de un área o persona independiente que maneje el SCI, manuales no diseñados, descono- cimiento de roles y responsabilidades de algunos órganos frente al control y la divulgación de las políticas, misión, visión y valores corporativos que impiden su funcionalidad y el verdadero objetivo de su implementación.

En este mismo sentido, para Arboleda, Sánchez y Sanabria (2016), quienes llevaron a cabo el estudio titulado "Factores de éxito competitivo: caso ESAL Barranquilla", encontrando situaciones similares a las destacadas en la presente investigación aseguran que "es importante resaltar los canales de comunicación existentes en las organizaciones que permitan un flujo efectivo de la información y generar un escenario de confianza" (p.174). Esto conllevaría al fortalecimiento de la relación del personal vinculado a la organización con el SCI y de esta manera contemplar la utilidad y dinámica del mismo dentro de las organizaciones.

\section{Estructuración, documentación y funcionamien- to del SCI bajo los componentes del modelo de control interno COSO}

Este modelo es muy completo y examina cada una de las áreas de la empresa con el fin de analizar y relacionar cada uno de los factores que puedan afectar o representen un riesgo para el negocio o desarrollo del objeto social, el cual tiene como función principal:

Reconocer la existencia de procesos, de un personal que los lleva a cabo (...), de una seguridad razonable para la administración y el Consejo de directores con miras a la consecución de los objetivos (operacionales, información financiera y de cumplimiento) y también la existencia de unos componentes interrelacionados (López y Guevara, 2016, p. 5).

Por lo tanto, a través de esta herramienta se busca la eficacia y eficiencia de las operaciones, fiabilidad de la información financiera, y cumplimiento de las leyes y normas que sean aplicables. Para lograr estos fines y realizar un mejor control y análisis de la compañía, los componentes básicos que contempla este modelo y que permiten desarrollar el control interno se basan en: el ambiente de control Evaluación del riesgo, información y comunicación, actividades de control y Monitoreo y seguimiento. 


\section{Ambiente de Control}

A través del ambiente de control se busca proporcionar una buena relación del recurso humano con la herramienta, produciendo un entorno en el cual se estimule y se permita la correcta implementación de los controles, así como la existencia de las condiciones para llevar a cabo la aplicación de los mismos; todo esto puede verse afectado cuando no existe una estructura organizacional adecuada, tal como se pudo observar en la presente investigación, toda vez que existen cargos y roles documentados pero que no se encuentran visibles dentro del organigrama. Bajo el mismo componente, se puede observar que, las promociones laborales son muy escasas, debido al nivel de exigencia y responsabilidad para pasar a otra área, pero cuando sucede se deja constancia de ello en los respectivos libros de actas; en cuanto a las medidas correctivas, no se lleva un libro o manual diario donde estas sean anotadas sino que se realizan de forma verbal por cada jefe de área y cuando la ocasión lo amerite se lleva ante instancias superiores, que son quienes determinan el proceso a seguir, dejando constancia de ello en las respectivas actas; por último, se puede decir que existe poca rotación de personal en las áreas, lo cual indica que la Fundación garantiza la estabilidad y las buenas condiciones laborales de su personal.

Los autores Duarte y Beltrán (2016) obtuvieron resultados similares en su estudio "Implementación de mejoras en los procedimientos contables y administrativos para la Fundación Recogiendo Semillitas", donde identificaron además, que existen factores tales como: la carencia de un organigrama, falta de segregación de funciones, ineficiencia de los procesos y la falta de elaboración de manuales, que son cruciales determinar en la aplicación del ejercicio del control y mejora.

\section{Evaluación del riesgo}

La evaluación del riesgo es un tanto compleja y necesaria, ya que ello implica tener la capacidad y disponibilidad para tomar acciones preventivas, de detección o correctivas en el momento preciso, logrando un mejoramiento del control. Uno de los puntos cruciales en este apartado es la dirección de las prácticas administrativas, contables y financieras bajo las políticas establecidas.
Se pudo evidenciar a través del Check List realizado que no existe como tal un manual de procesos y procedimientos sino que se encuentran estos incluidos en un archivo denominado Manual de Campo o PFM 2.5, al igual que las políticas y procedimientos organizacionales. Además, se encontró que, en la mayoría de casos las prácticas administrativas y financieras se llevan acorde a lo establecido en las políticas, sin embargo, existe un riesgo alto en cuanto a la información contable, ya que al no llevarse esta de forma continua y bajo un sistema contable se dificulta la obtención de información de forma oportuna y eficaz que permita tomar decisiones en un momento dado, ya sea con el fin de verificar el alcance de los objetivos y metas establecidas o tomar medidas preventivas y/o correctivas en cuanto al funcionamiento de la entidad, representando también esto un riesgo a nivel legal, toda vez que incurre en el incumplimiento a lo dispuesto en el artículo 45 de la ley 190 de 1995 en el cual se estipula la obligatoriedad de las ESAL de llevar contabilidad y las diversas sanciones a que se encuentra expuesta.

De acuerdo a los resultados revelados, en estudios similares se pudo establecer que existen factores claves para lograr el éxito de las organizaciones, dentro de los cuales se puede destacar la evaluación y mitigación de los riesgos, teniendo en cuenta todos aquellos estándares normativos y operativos, lo cual conlleva a una mejor calidad y confiabilidad por parte de los usuarios (Rodríguez y Suárez, 2014). Es decir, que es necesario llevar a cabo dentro de las entidades acciones de evaluación y mitigación del riesgo, dentro de los cuales se encuentra aquel de tipo legal.

\section{Actividades de Control}

Dentro del componente de actividades de control se buscan desarrollar acciones que garanticen la minimización de los riesgos y la consecución de los objetivos, trayendo consigo la implementación de ciertas estrategias por parte de la administración, lo cual sucede de acuerdo con Cárdenas, Rincón y Guio (2017) porque "la empresa alcanza un nivel de complejidad tal, que la obliga a generar estrategias y a buscar un modelo de control de las mismas" (p. 2). Esto refleja que una de una de las más usadas es la formulación de los manuales de procesos, procedimientos y políticas, los cuales deben pasar por un constante sistema de actualización para que puedan dar res- 
puesta a todas las actividades. Es decir, para que se puedan llevar a cabo las actividades de control es indispensable que las organizaciones, implementen y fortalezcan dentro de sus estrategias la actualización e innovación constante, asegurando con ello el éxito y perdurabilidad en el tiempo. Se pudo identificar que dentro del archivo que lleva la Fundación denominado Controles Internos y en el Manual de Campo o PFM 2.5 donde se lleva la documentación de procesos y procedimientos hace falta la descripción de algunos; otros por su parte, no han sido actualizados y alineados a los nuevos marcos normativos, legales y organizacionales.

\section{Información y Comunicación}

La información y comunicación es esencial, ya que ayuda al mejoramiento de los procesos y permite brindar una mayor seguridad, conocimiento y confiabilidad en el negocio u objeto social, tanto a los colaboradores como a los clientes de la información externos. De acuerdo a las observaciones consignadas se pudo evidenciar que SI se realiza una recopilación de información de terceros bajo las políticas y procedimientos establecidos, sin embargo, no se lleva una base de datos en cuanto a esta información de agentes externos. De igual manera, se pudo verificar que no existen unos plazos establecidos para la recopilación, divulgación y respuesta a las comunicaciones internas y externas, lo cual puede derivar en una dirección y gestión inadecuada e ineficaz de la organización y puede generar traumatismo y sobrecarga laboral, así como demora en la presentación de información a terceros; asimismo, se pudo conocer que la fundación cuenta con una página web registrada, sin embargo no existe claridad sobre la persona responsable de su manejo, lo cual ocasiona que esta no se encuentre actualizada, conociendo que a través de ella puede extenderse y darse a conocer ante un mayor público.

Por otro lado, la información que maneja la Fundación del personal y de terceros (de acuerdo a los criterios de medición) se encuentra en un nivel MEDIO ya que se garantiza la seguridad y acceso solo al personal autorizado, sin embargo, existen documentos que presentan una seguridad BAJA ya que se encuentran a la vista del público $\mathrm{y}$ en algunos casos no funciona el sistema de seguridad en el cual se encuentran ubicados, lo cual puede ocasionar robo y pérdida de documentos e información de agentes internos y externos. De igual manera, existen algunos servidores y equipos que contienen información legal y acceso a cuentas de la entidad, pero no tienen restricciones de acceso al iniciar sesión en los mismos. El marketing juega un papel fundamental a la hora de llegar a la satisfacción de consumidor; de tal manera, la publicidad es un tema fundamental ya que es sensible a cometer error en el mercado. (Suárez y Salazar, 2016)

Como apoyo a lo anterior, en el estudio "Fortalecimiento Empresarial a Entidades sin ánimo de lucro. Fusagasugá - región" realizado por Escobar, Ruiz y Escobar (2015) obteniendo resultados similares en cuanto al componente de información y comunicación, argumentan que "existe la necesidad de fortalecer la capacidad endógena institucional para la comunicación y el desarrollo competitivo de la economía, la tecnología y el emprendimiento" (p.101). Esto permite deducir, que uno de los puntos quizás más débiles dentro de este tipo de organización como las ESAL (aunque se puede dar también en otras organizaciones), son sus sistemas de información, toda vez que son vulnerables y poseen muy poca seguridad en su manejo. De igual forma, Pacheco (2017), en «El gravamen a los movimientos financieros: análisis del recaudo tributario versus la oferta monetaria en Colombia», empleando una metodología menos sofisticada por medio de análisis de tendencias de los principales agregados monetarios y el comportamiento del tributo a lo largo de su entrada en vigencia.

\section{Supervisión y Monitoreo}

El resultado de la implementación y ejecución de este componente se traduce en un mayor nivel de calidad que conecta varios procesos. La realización de actividades de supervisión y monitoreo no corresponde a una sola área o departamento, sino que hace parte de la responsabilidad de cada persona para la consecución de los objetivos. Desde este enfoque "La supervisión identifica si las acciones de cada nivel del proceso conducen a los otros niveles previstos o si es necesario hacer cambios. El monitoreo permanente incluye actividades de supervisión, directamente por las distintas estructuras de dirección" (Vega y Nieves, 2016, p. 4). Por consiguiente, se puede inferir que para el caso del componente de supervisión 
y monitoreo, existe una interrelación y transversalidad entre cada proceso, área y actividad de la empresa, tal que si ocurre algún suceso negativo en uno de ellos, eso crea una cadena o línea de daño general si no se corrige a tiempo.

A través de los diferentes instrumentos de recolección de datos se pudo obtener que, aunque existen controles internos en la entidad estudiada, es necesario realizarle algunas mejoras y ajustes al mismo que permitan que este sea eficiente, ajustes tales como mayor documentación de las actividades, identificación y responsabilidades de la persona responsable del control interno, así como el diseño, actualización y/o implementación de los diferentes manuales que ayudarán a crear un mayor nivel de productividad, eficiencia y eficacia en las actividades.

\section{Principales recomendaciones para fortalecer el Sistema de Control Interno y mejorar la toma de decisiones en los procesos administrativos, contables y financieros}

Luego de realizar el proceso de verificación o Check List del control interno se identificó que no existe un manual de funciones y responsabilidades sino que estas se encuentran descritas en los respectivos contratos. Del mismo modo, se obtuvo que el $100 \%$ de los encuestados, tienen una percepción ALTA sobre la filosofía de dirección y el estilo de gestión desarrollado, demostrando, que el trabajo realizado por la gerencia, directivos y jefes de área tiene una buena aceptación dentro del resto de personal. Sin embargo, se hace necesario en este sentido, expresar que es recomendable se implemente y documente una ruta y sus respectivos responsables para realizar las quejas, sugerencias, recomendaciones y/o denuncias por parte del personal interno y externo, logrando una mayor transparencia y evaluación de la gestión desarrollada. Si el liderazgo presenta fallas trasciende en un errado direccionamiento hacía sus colaboradores originando desalineación, confusión en sus funciones, obteniendo resultados perjudiciales, ocasionando un alto grado de desmotivación, además de un clima organizacional tenso e inapropiado que no permite el trabajo en equipo en la empresa (Rivera \& Conrado, 2016, pág. 64)

Luego de realizar las observaciones respectivas se pudo establecer que la entidad cuenta con adecuado número de personal para el nivel de ac- tividades y operaciones desarrolladas; en cuanto a la ubicación geográfica se podría decir que esta es pertinente para la labor y zona de impacto; mientras tanto, el impacto social se puede decir que en el último año no ha tenido un crecimiento significativo, sino que contrario a esto ha disminuido la cobertura y nivel de patrocinio. Por último, los datos obtenidos revelan que el $83 \%$ de los encuestados considera el nivel de su actual condición laboral de acuerdo a sus conocimientos, habilidades y competencias, como ALTO, entretanto, que el $17 \%$ restante considera que este nivel es MEDIO. Esto deja entrever que si existiera una mejor condición laboral en otra organización las personas optarían por tomar la decisión de tomar las respectivas ofertas, lo cual va muy ligado al tema de calidad laboral, donde se pudo constatar que el $82 \%$ de las contrataciones corresponden a la modalidad de Prestación de servicios mientras que el otro $18 \%$ corresponden a la modalidad de Contrato Indefinido, coexistiendo con ello una mayor incertidumbre con respecto al futuro y condiciones laborales.

\section{Conclusiones}

Frente a lo expuesto anteriormente, se puede argumentar que, el tema de fortalecimiento del SCI que cada entidad, empresa u organización tiene implementado, independientemente del modelo seguido, es una responsabilidad que aplica e involucra esencialmente a las partes superiores de la estructura organizacional, ya que son ellos quienes desarrollan la misión y visión de la entidad, para que luego se trabaje en las diferentes estrategias y la trazabilidad de los objetivos y metas.

Es necesario que exista un área o persona encargada del control interno, y que en lo posible este no sea manejado por la gerencia y la administración. Así mismo, el diseño de un código de ética es esencial dentro de las organizaciones, esencialmente cuando nos referimos a las ESAL, ya que su inexistencia puede afectar la conducta y toma de decisiones de una persona frente a determinadas situaciones, ya que no existe claridad bajo que parámetros actuar. Igualmente, es pertinente que pueda existir un Comité de auditoría u órgano que cumpla las funciones de este, velando por el cumplimiento de las diferentes políticas y prácticas establecidas, de tal forma que se minimice en cada acción los riesgos; brindando 
también apoyo cuando este sea necesario para superar alguna situación, para lo cual se requiere que estén debidamente familiarizados y actualizados con las operaciones y actividades que lleva a cabo la entidad.

La divulgación de la misión, visión y valores corporativos, hace parte de las actividades empresariales, buscando a través de esto, un mayor reconocimiento y posicionamiento en la comunidad, que es sin duda, uno de los principales ejes que busca alcanzar cualquier organización, especialmente las ESAL, ya que esto trae consigo el beneficio de una mayor concentración local y la fijación de la mirada de muchas organizaciones nacionales, internacionales, públicas y privadas. Por tal motivo, también es necesario que la descripción de algunas políticas, procesos y procedimientos sean actualizadas y alineadas a los nuevos marcos normativos, legales y organizacionales.

Cuando se realiza recopilación de información, ya sea interna o de terceros, estos se deben hacer bajo las políticas y procedimientos establecidos, llevando conjuntamente una base de datos, garantizando la seguridad y acceso a esta solo al personal autorizado.

En cualquier organización, siempre deberán existir plazos establecidos para la recopilación, divulgación y respuesta a las comunicaciones internas y externas, con el fin de no derivar en una dirección y gestión inadecuada e ineficaz de la organización y se pueda evitar generar traumatismo y sobrecarga laboral, así como demora en la presentación de información a terceros.

La contabilidad es esencial dentro de cualquier organización, y aunque no represente en la misma uno de los procesos más relevantes e importantes, es necesario Dar cumplimiento a lo dispuesto en los respectivos marcos normativos que rigen su actividad. Teniendo en cuenta de igual manera que representa un factor importante al momento de tomar decisiones e implementar mejoras dentro de la organización, sirve igualmente como medio de control de las operaciones.

A raíz de todo esto, se puede decir que, el nivel de estructuración del SCI es débil, lo cual se obtuvo a través de diferentes ítems de la lista de verificación empleada; también se pudo evidenciar que este sistema se encuentra documentado, pero no posee las actualizaciones y revisiones adecuadas de acuerdo a los lineamientos y normas vigentes; de esta manera, se puede argumentar que el funcionamiento se puede clasificar en el nivel de NO APTO o INADECUADO, atendiendo a las descripciones y formulaciones anteriores.

\section{Referencias}

Aguiar, H. (1996). El fraude en las organizaciones. Contaduría, 28 (1), 193-208

Arboleda, G.B., Sánchez, A.M. y Sanabria, N.J. (2016). Factores de éxito competitivo: caso ESAL Barranquilla. Dimensión Empresarial, 14(2), 163-179. doi: http://dx.doi.org/10.15665/rde.v14i2.740

Arens, A.A., Elder, R.J., y Beasley, M.S. (2007). Auditoría un enfoque integral. México D.F., México: Pearson Educación.

Cárdenas, M., Rincón, N., y Guio, M. (Septiembre de 2017). La contabilidad de dirección estratégica, información suficiente para decisiones pertinentes. En U. Militar de Nueva Granada (Presidencia), Jornada Internacional de Estudios Disciplinares en Contabilidad. Conferencia llevada a cabo en el congreso II Jornada Internacional de Estudios Disciplinares en Contabilidad y II Encuentro de Estudiantes de Contaduría Pública, Bogotá, Colombia.

De la Cruz, S. (2018). Teorías de desarrollo económico y desarrollo económico regional: un enfoque conceptual, 7(7), p. 76-84. https://doi.org/10.21803/adgnosis.v7i7.295

De la Dehensa, G. (2000). Comprender la Globalización. Recensiones Revista Del Ministerio de Trabajo y Asuntos Sociales 31 (1), 165-169.

Duarte, A., y Beltrán, D. (2016). Implementación de mejoras en los procedimientos contables y administrativos para la Fundación Recogiendo Semillitas (tesis de pregrado). Universidad de La Salle, Bogotá D.C., Colombia. 
Escobar, E.A., Ruiz, M., y Escobar, G. (2015). Fortalecimiento Empresarial a Entidades sin ánimo de lucro. Fusagasugá - región. Pensamiento y Gestión, 39(1), 86-101.

Estupiñán, G. R. (2015). Administración de riesgos e.r.m. y la auditoría interna (2a. ed.). Bogotá, Colombia: Ecoe Ediciones.

Hernández, R., Fernández, C., y Baptista, M. (2010). Metodología de la investigación. México D.F, México: Mc-Graw Hill.

López, O.R. y Guevara, J.A.(2016). Control Interno en Colombia: Un diagnóstico desde lo teórico. En-Contexto Revista de Investigación en Administración, Contabilidad, Economía y Sociedad, 4(5), 1-24.

Mantilla, S. (2008). Auditoría Financiera de PYMES. Bogotá, Colombia : Ecoe Ediciones.

Mejía, J. (2000). El muestreo en la investigación cualitativa. Investigaciones sociales, 4(5), 165-180.

Pacheco Martínez, G. A. (2017). El gravamen a los movimientos financieros: análisis del recaudo tributario versus la oferta monetaria en Colombia. Revista ADGNOSIS, 6(6), 177-193. https://doi.org/10.21803/adgnosis.v6i6.198

Pérez, G. (1994). Investigación Cualitativa. Retos e Interrogantes. Madrid, España: Editorial La Muralla S.A.

Rivera Cerpa, Y. M., \& Conrado Tobón, J. (2016). Impacto de los líderes en la productividad de las empresas de servicio de aseo en la ciudad de Barranquilla. Dictamen Libre, (19), 57-68. https://doi.org/10.18041/2619-4244/d1.19.2901

Rodríguez, G., y Suárez, L. (2014). Análisis del modelo actual de gestión administrativa y de los factores determinantes del éxito empresarial en la Cooperativa San Pio X de Granada Ltda., “COOGRANADA” (Tesis de maestría). Universidad de La Salle, Bogotá D.C., Colombia.

Suárez, S. y Salazar, B. (2016). El marketing experiencial como herramienta para el fortalecimiento de la imagen corporativa de las empresas del sector comercial colombiano. Revista Dictamen Libre, (19), 97-109. https://dialnet.unirioja.es/servlet/articulo?codigo=6104141

Vega, L. y Nieves, A.F. (2016). Procedimiento para la Gestión de la Supervisión y Monitoreo del Control Interno. Ciencias Holguín, 22 (1), 1-19.

Villasmil, M. y Polo, F. (2018). Estudio comparado sobre la regulación del impuesto al valor agregado (IVA) en Colombia y España. Dictamen Libre, 23, 11-31. https://doi.org/10.18041/26194244/d1.23.5142 